\title{
Characterization and remediation of sample index swaps by non-redundant dual indexing on massively parallel sequencing platforms
}

Maura Costello* (1), Mark Fleharty, Justin Abreu, Yossi Farjoun, Steven Ferriera, Laurie Holmes, Brian Granger, Lisa Green, Tom Howd, Tamara Mason, Gina Vicente, Michael Dasilva, Wendy Brodeur, Timothy DeSmet, Sheila Dodge, Niall J. Lennon and Stacey Gabriel

\begin{abstract}
Background: Here we present an in-depth characterization of the mechanism of sequencer-induced sample contamination due to the phenomenon of index swapping that impacts Illumina sequencers employing patterned flow cells with Exclusion Amplification (ExAmp) chemistry (HiSeqX, HiSeq4000, and NovaSeq). We also present a remediation method that minimizes the impact of such swaps.

Results: Leveraging data collected over a two-year period, we demonstrate the widespread prevalence of index swapping in patterned flow cell data. We calculate mean swap rates across multiple sample preparation methods and sequencer models, demonstrating that different library methods can have vastly different swapping rates and that even non-ExAmp chemistry instruments display trace levels of index swapping. We provide methods for eliminating sample data cross contamination by utilizing non-redundant dual indexing for complete filtering of index swapped reads, and share the sequences for 96 non-combinatorial dual indexes we have validated across various library preparation methods and sequencer models. Finally, using computational methods we provide a greater insight into the mechanism of index swapping.

Conclusions: Index swapping in pooled libraries is a prevalent phenomenon that we observe at a rate of 0.2 to $6 \%$ in all sequencing runs on HiSeqX, HiSeq 4000/3000, and NovaSeq. Utilizing non-redundant dual indexing allows for the removal (flagging/filtering) of these swapped reads and eliminates swapping induced sample contamination, which is critical for sensitive applications such as RNA-seq, single cell, blood biopsy using circulating tumor DNA, or clinical sequencing.
\end{abstract}

Keywords: Next generation sequencing, Massively parallel sequencing, ILLUMINA sequencing, Index swapping, Index hopping, Multiplexing, Barcodes, Index, Indexes, Exclusion amplification

\footnotetext{
* Correspondence: costello@broadinstitute.org

Broad Genomics, Broad Institute of MIT and Harvard, 320 Charles Street,

Cambridge, MA 02141, USA
}

(c) The Author(s). 2018 Open Access This article is distributed under the terms of the Creative Commons Attribution 4.0 International License (http://creativecommons.org/licenses/by/4.0/), which permits unrestricted use, distribution, and reproduction in any medium, provided you give appropriate credit to the original author(s) and the source, provide a link to the Creative Commons license, and indicate if changes were made. The Creative Commons Public Domain Dedication waiver (http://creativecommons.org/publicdomain/zero/1.0/) applies to the data made available in this article, unless otherwise stated. 


\section{Background}

"As sequencing costs decline..." is a common phrase in the field of genomics as rapid advances in massively parallel sequencing platforms are making population scale sequencing a reality. In 2015, Illumina introduced the HiSeqX sequencer, utilizing their newest patterned flow cell and ExAmp chemistry technologies $[1,2]$. These instruments were purpose-built human whole genome machines, capable of producing $1000 \mathrm{~Gb}$ of data per flow cell and cutting sequencing costs by two thirds over previous models. Soon after, Illumina released the HiSeq 4000 \& 3000 instruments, utilizing that same patterned flow cell technology but allowing for sequencing of a wider variety of library preparation types. NovaSeq, their newest sequencer released in mid 2017, utilizes this same ExAmp chemistry with patterned flow cells but promises even higher yields of up to 3 terabases of data per flow cell by 2018 [3].

Sequencing biological samples at scale requires a highly streamlined workflow that makes the most efficient use of instrument yield and eliminates effects of lane to lane variability. To achieve the maximum cost efficiency, sample multiplexing on sequencer has become a necessity even for whole genomes. However, as others have recently reported [4-8], this new ExAmp chemistry can lead to data integrity issues due to the phenomenon of index switching or swapping. Illumina and others have reported that this swapping is likely due to residual excess free primer or adapters in the samples that, when pooled and mixed with the ExAmp reagents, can lead to spurious extension of library fragments with an oligo containing the wrong sample index. When single or combinatorial dual index schemes are used, these swapped indexes lead to read mis-assignment and can manifest as cross-contamination within a pool $[4,5]$.

As this phenomenon is a property of the flow cell chemistry itself and will occur to some degree in every HiSeqX, HiSeq 4000, or NovaSeq sequencing run, the only options to completely eliminate the effects of index swapping on data integrity are to sequence one sample per lane or to use a nonredundant dual indexing strategy. As running one sample per lane is not financially feasible at scale, we needed a suitable dual index scheme. However, as of mid 2017, most vendors including Illumina still did not have a full plate, non-redundant dual indexing solution available. To create the ability to pool entire 96 well plates of dual indexed libraries, we have designed and implemented a unique dual indexing method utilizing a set of non-redundant indexes (comprising 96 unique "i7" and 96 unique "i5") that we have validated across multiple sample preparation workflows and Illumina sequencer models, including the NovaSeq.

\section{Results}

Index swapping of PCR-free genomes on HiSeqX

We began multiplexing our PCR-free human whole genome libraries prior to sequencing on HiSeqX in 2015, starting with pools of 8 in February and then eventually pools of 24 by November. In our workflow, data from multiple lanes for each library are aggregated together after sequencing to achieve the desired 30X human genome coverage, and downstream analysis is performed on the aggregated data file. We chose to pool prior to sequencing for multiple reasons: (1) ease of workflow; (2) improved consistency of lane loading; and (3) reduction in the effects of lane to lane performance variability we had observed with HiSeqX flow cells. Following implementation of pooling, we began to get reports from our data users that they were observing elevated rates of sample contamination in aggregated PCRfree genome data. We ran all aggregated sample BAM (Binary Alignment/Map) files generated during the past year on HiSeqX through "VerifyBamID", a tool designed to estimate sample \% contamination in human sequencing data [9] and confirmed widespread sample contamination in PCR-free libraries at an average of $1.2 \%$. This contamination appeared to be present in all samples generated from pooled sequencing, from all tissue sources, projects and collaborators, and appeared to worsen as we switched from 8-plex to 24-plex pools (Fig. 1).

At the same time, we were also processing a smaller number of PCR-plus whole genomes on the HiSeqX, and while we did not observe contamination at the same magnitude as PCR-free, contamination in PCR-Plus libraries had also increased significantly during the same time period (Fig. 1). Our PCR-free and PCR-plus genome libraries are made using identical protocols in the lab with the same reagents and adapter plates, by the same team, and on the same pieces of automation; the only difference is the addition of 8 cycles of PCR at the end of the process for PCR-plus. The difference in contamination rates between these two workflows provided some evidence that the contamination was likely not coming from the library preparation processes. We then took two different 24-plex PCR-free genome pools with mean library contamination rates from HiSeqX of $6.40 \%$ and $1.65 \%$ respectively, and re-sequenced these pools on MiSeq where we observed the rates of contamination for both pools dropping to $0.60 \%$ and $0.09 \%$, respectively. This 10-fold discrepancy in contamination rate between the HiSeqX and MiSeq for the same pool of libraries strongly indicated that the contamination event was happening during the ExAmp patterned flow cell preparation or sequencing process. This observation has subsequently been reported by others [4-8]. 


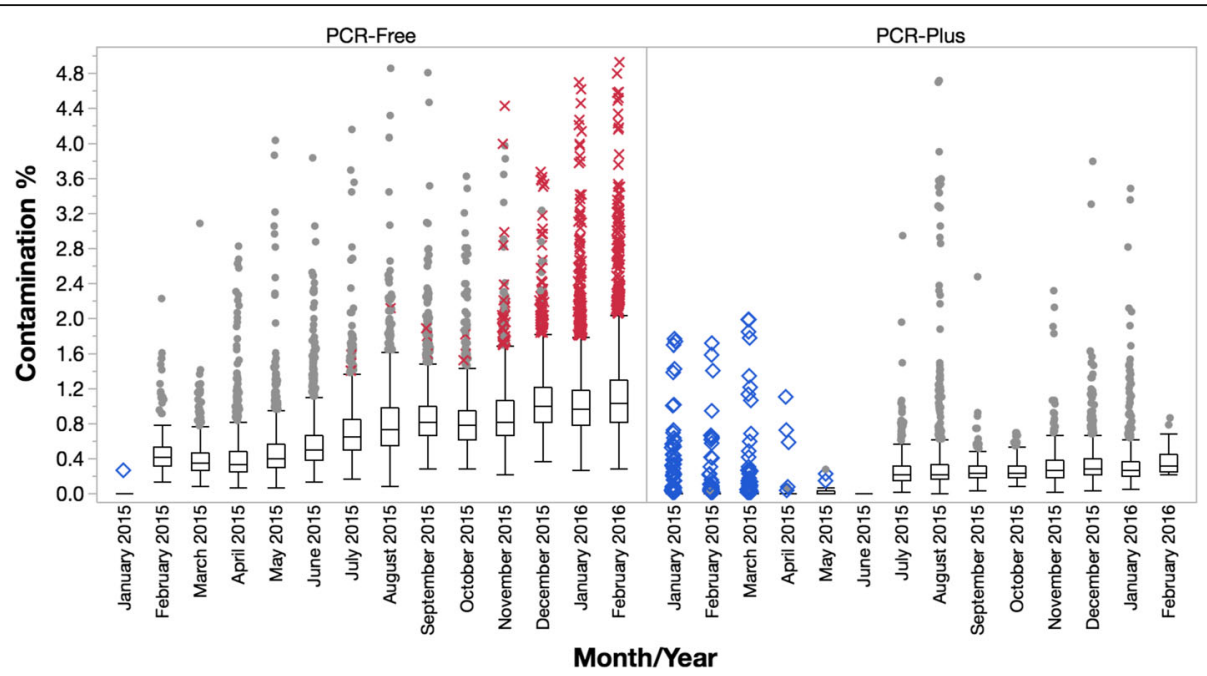

Fig. 1 Percent contamination over time for whole genomes sequenced on HiSeqX. Panel at left: Single indexed PCR-free library contamination by month. Contamination significantly increased when we began 8-plex pooling and worsened as we introduced 24-plex pooling. Panel at right: Single indexed PCR-plus library contamination by month. Although overall contamination was lower for PCR-plus, rates did increase significantly as well when we began pooling

\section{Non-redundant dual indexing enables identification \& filtering of index swapped reads}

When first released, the HiSeqX was only configured to read a single i7 library index [2]. In order to characterize what was causing this contamination, we enabled dual indexing on the HiSeqX by altering the sequencing recipes and supplementing the required i5 dual index sequencing primer. Our genome adapters were designed to be dual indexing enabled, therefore we were able to sequence a set of four 2-plex library pools containing unique combinations of dual indexes on the altered HiSeqX reading both the $i 7$ and 15 indexes. We then ran demultiplexing and analysis on the same set of read groups from the same flow cell two different ways: first, using just the i7 single index data and second, using both the i7 and i5 dual index reads. In the data demultiplexed using just the i7 index, the contamination averaged $0.89 \%$; however, in the data demultiplexed using both i7 and i5 indexes, the mean contamination rate dropped to just $0.13 \%$ (Additional file 1: Figure S1). Examining the non-demultiplexed reads from the dual indexing analysis, we observed an unusually high number of high quality reads (Q30 or greater) where the i7 and i5 indexes weren't in the expected combinations from our standard adapter set. However, within this population of index mismatched index reads, we observed only indexes originating from libraries within the pool itself and no significant sources of outside index contamination, further indicating this wasn't a random contamination event happening during the lab processes but something constrained to within the pool itself.

We concluded that indexes from library fragments from one sample were replacing other samples' indexes within pools during the HiSeqX ExAmp process, which has also been confirmed by other teams [4-8]. We further hypothesize that contamination rates for single index libraries escalated as the number of samples in the pool increased because increasing the number of different libraries in an ExAmp reaction also increases the likelihood that a given library fragment will swap with a different sample's library fragment rather than selfswapping. Unlike some [10], we have subsequently observed indexing swapping in all runs sequenced on ExAmp patterned flow cell instruments including the HiSeqX, HiSeq 4000, and NovaSeq, which agrees with Illumina's own documentation that any sequencer model that relies on ExAmp and patterned flow cells can and will swap indexes [5]. While efficient clean up steps to remove residual index oligos may help reduce the rate of indexing swapping and computational methods may be able to compensate to some degree [11], utilizing unique non-redundant dual indexing is the only way to truly filter out swapped reads from pools sequenced on patterned flow cell data [5].

We have created and refined a novel set of 1928 base pair indexes, which can enable up to a 96 nonredundant dual index combinations (Additional file 2). This set has been validated in various sample preparation methods, and performs robustly both when used in adapter oligos ligated during library construction and in PCR primer formats that allow addition of dual indexes in targeted PCR and Nextera based protocols. They have also been screened across all Illumina sequencing platforms including random clustering and ExAmp, and 2-color or 3-color imaging, including the NovaSeq. As of September 2017, we have used this set of dual 
indexes on over 150,000 whole exomes on MiSeq, HiSeq 2500 , and HiSeq 4000, and over 57,000 genomes on dual index enabled HiSeqX instruments, with average contamination rates close to zero (Fig. 2).

\section{Measuring swap rates across various sample preparation methods and sequencers}

As we had observed a clear difference between PCR-plus and PCR-free genome contamination rates on HiSeqX (due to swaps), we wished to survey the swap rates across our major library construction methods and across both random cluster amplification based sequencers and ExAmp patterned flow cell sequencers.

The results in Table 1 demonstrate that different library preparation methods can have drastically different swap rates and that random cluster amplification sequencers like MiSeq, NextSeq, or HiSeq 2500 can also introduce a baseline level of index swapping, although the rate is generally low [12].

While others have reported upwards of a $10 \%$ rate of index swapping on patterned flow cell sequencers [4], the highest rate of swapping we have observed is $\sim 6 \%$ in our PCR-free workflow. Swapping at that magnitude is rare and typically we have averaged $\sim 3 \%$ in PCR-free and $\sim 0.25 \%$ in PCR-plus genomes. We hypothesize that PCR-free whole genomes have the highest rate of swapping due to the low yield of this particular library type following library construction. While PCR amplified libraries typically need to be diluted between 20-fold to 200 -fold prior to sequencing depending on post-PCR yield, PCR-free libraries are diluted very little if at all as typical yields from that method are in the low nanomolar range. This lack of dilution likely leads to a higher ratio of free adapter to library fragments in PCR-free pools, increasing the chance that free adapter molecules will interact with library fragments during ExAmp chemistry.

Interestingly, the swap rates for both germline and somatic exomes are elevated even when pools are sequenced on MiSeq. This is due to index swapping occurring during the exome capture process itself. We pool up to 12 libraries per exome hybridization reaction. Following streptavidin bead immobilization, the captured DNA is amplified off the beads in a PCR reaction. It is in this multiplex PCR where index swapping is occurring, and we have observed this previously in exome capture methods from both Agilent (Additional file 1: Figure S2) and Illumina (Table 1), even when sequenced on MiSeq or other random clustering sequencers.

While the average rate of swapping in our RNA-seq data was relatively low at just $0.32 \%$, the effects of read misassignment in RNA-seq data can be readily apparent during downstream analysis. To evaluate a new RNA fusion caller, we had generated data using single indexed RNA-seq libraries on HiSeq 4000 from cell lines with known fusion events, including $\mathrm{K} 562$ containing the BCR-ABL1 fusion. In analysis, we detected reads from BCR-ABL1 fusion transcripts in the data files for all 3 cell lines tested, though only K562 should have that translocation (Fig. 3). This example highlights the danger of processing samples without unique dual indexing. Despite implementing sound lab practices to reduce residual adapter or primer, even methods with less than $1 \%$ indexing swapping could still generate spurious results in downstream data analysis.

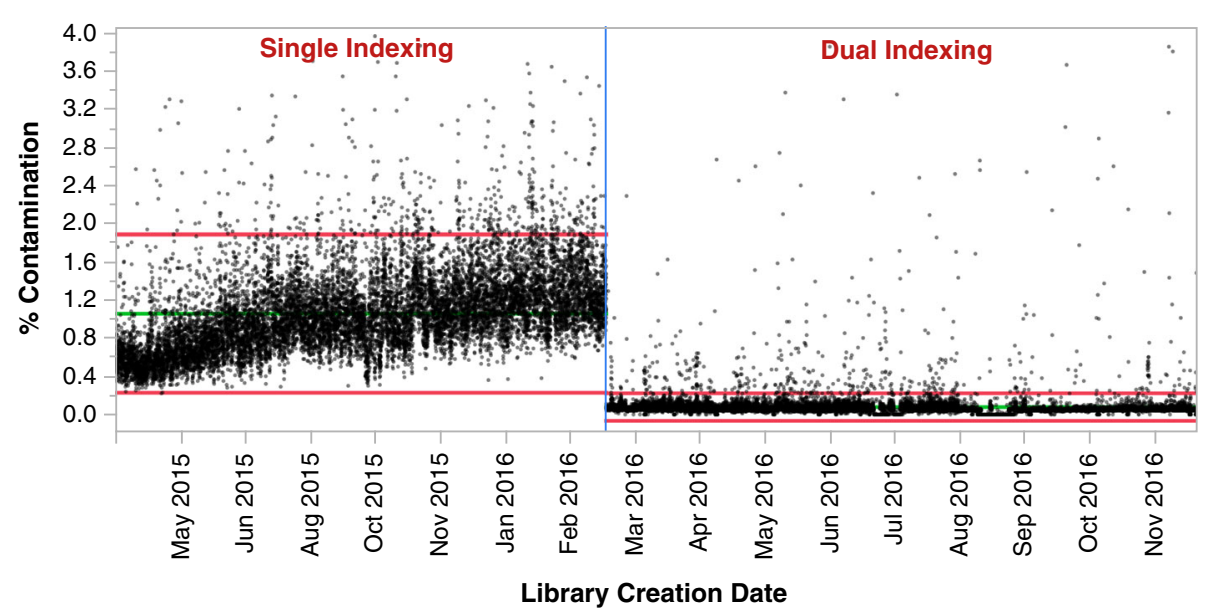

Fig. 2 Contamination for single versus dual indexed pooled PCR-free libraries on HiSegX. Percent contamination month by month continuous run chart as measured by VerifyBamID [3] for 24-plexed PCR-free genomes, demonstrating the drop in mean contamination after implementation of unique dual indexing. Red reference line is the mean, green reference lines are upper and lower control limits of the data generated by JMP statistical software 
Table 1 Mean Index swapping rates by library prep method and machine type

\begin{tabular}{|c|c|c|c|c|c|c|}
\hline \multirow[t]{2}{*}{ Library type } & \multirow[t]{2}{*}{ Method description } & \multirow{2}{*}{$\begin{array}{l}\text { Multiplex PCR prior } \\
\text { to sequencing? }\end{array}$} & \multirow{2}{*}{$\begin{array}{l}\text { Mean library } \\
\text { yield }\end{array}$} & \multicolumn{3}{|c|}{ Index swap rate (\%) ${ }^{a}$} \\
\hline & & & & $\overline{\text { MiSeq }}$ & HiSeqX or 4000 & NovaSeq $^{b}$ \\
\hline PCR-free genomes & DNA shearing + adapter ligation & - & $2.8 \mathrm{nM}$ & $0.13 \pm 0.08$ & $3.01 \pm 0.91$ & $4.85 \pm 0.88$ \\
\hline PCR-plus genomes & DNA shearing + adapter ligation & - & $141.1 \mathrm{nM}$ & $0.03 \pm 0.04$ & $0.24 \pm 0.06$ & no data \\
\hline Somatic exome & DNA shearing + adapter ligation & + & $354.2 \mathrm{nM}$ & $0.67 \pm 0.08$ & $0.83 \pm 0.23$ & $0.52 \%$ \\
\hline Germline exome & Nextera transposase & + & $286.2 \mathrm{nM}$ & $0.49 \pm 0.12$ & $0.68 \pm 0.19$ & no data \\
\hline Stranded mRNA & cDNA prep + adapter ligation & - & $39.8 \mathrm{nM}$ & $0.01 \pm 0.001$ & $0.32 \pm 0.02$ & no data \\
\hline
\end{tabular}

${ }^{a}$ All swap rate values for each library type are means of 8 different pool \& flowcell observations

${ }^{\mathrm{b}}$ As of submission, NovaSeq data had only been generated for PCR-free genomes and one flow cell of exomes

Additionally, the standard deviation in swap rate data in Table 1 demonstrates that there can be variability of swap rates within a given library preparation method. To determine the source of this variation, we collected swap rate data on 24-plex PCR-free library pools that were sequenced across more than one flow cell, allowing us to measure variation in swap rate within an individual flow cell and across multiple flow cells containing the same pool. Index swap rates by pool and flow cell/lane are plotted in Fig. 4. Swap rates for the same pool of libraries typically stay consistent across lanes within a flow cell with occasional outlier lanes, but can vary greatly between different flow cells. This data indicate that the rate of index swapping for a given pool of libraries is not entirely driven by the amount of free adapter contained in that library pool, but rather can also be influenced by ExAmp reaction setup or reagents and flow cell lots.

\section{Characterizing the index swapping mechanism}

Utilizing unique dual indexing, we could now compare in depth swapped and unswapped reads with the goal of better understanding the mechanisms and kinetics of this phenomenon. First, we looked at a pool of 6 PCRfree dual indexed libraries sequenced on a lane of HiSeqX to determine: (i) if the rate of index swapping was the same across the entire flow cell from inlet to outlet, and (ii) if swapping occurred in a relatively normal distribution for each index or if there were biases for which indexes may be more likely to swap. The data showed that the rate of swapping was fairly uniform across both flow cell surfaces regardless of tile location (Fig. 5a) and that we observed all possible index swap combinations at a relatively uniform distribution around the mean, indicating swap rates are not likely driven by amplification biases for or against certain index barcodes sequences (Fig. 5b).

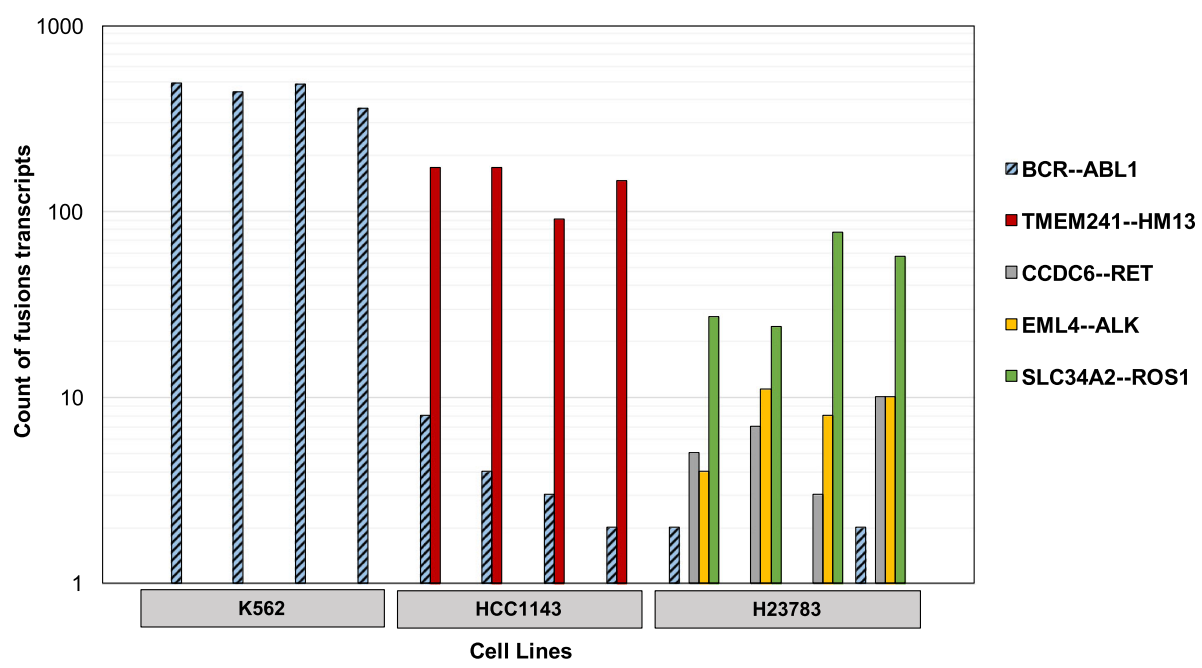

Fig. 3 Index swapping leads to incorrect assignment of reads from fusion transcripts in cell line RNA-seq data. Counts of reads spanning fusion transcripts for 5 different gene fusions in 3 different cell lines using STAR-Fusion software. Four RNA-seq libraries were pooled for each cell line for a total of 12 libraries, and sequenced on a HiSeq 4000 lane. Only the K562 cell line should contain the BCR - ABL1 translocation, however reads containing BCR - ABL1 (blue and black striped) were also found in data files for the other two cell lines due to index swapping 


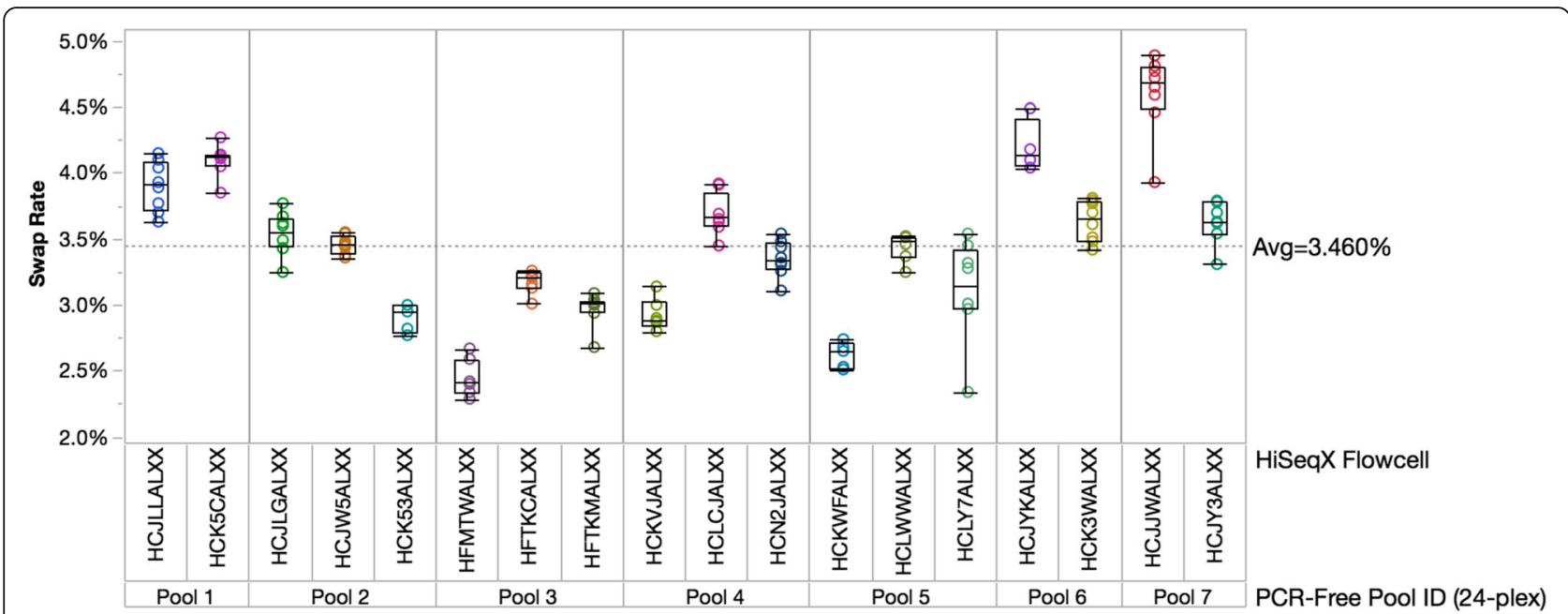

Fig. 4 Variability of index swap rates from pool to pool and flow cell to flow cell. Index swapping rates plotted for seven 24-plex pools, each sequenced on at least two HiSeqX flow cells and prepared using identical automated methods on a Hamilton MiniStar. Each data point represents a flow cell lane. The data shows variability between different pools, but also variability for the same pools run on different flow cells, indicating that flow cell and/or ExAmp reagents also influence swap rate variability

Next, we compared a variety of other sequencing and library metrics in both swapped and unswapped reads to determine if any correlations exist. We observed that swapped reads have smaller insert lengths and higher rates of chimerism than non-swapped reads (Fig. 5c), as well as tend to skew towards lower \% GC (Fig. 5d). These observations fit the hypothesis that swapping occurs during the ExAmp chemistry step, as shorter fragments with lower \%GC are known to amplify more efficiently in polymerase based amplification assays [13]. The increased rate of chimerism for swapped reads is of note for those wishing to perform structural variation detection, as these artefactual chimeric reads could be mistaken for reads derived from actual chromosomal rearrangements.

Finally, we pooled two libraries together with unique dual indexes: one made from human DNA and one from E. coli, which allowed us to more accurately measure the rates of swapping for each of the i7 and i5 ends of the library fragments (Table 2).

We observed that the i5 index was twice as likely to be swapped than the i7 index; however, the reason why one end of the adapter construct would preferentially be swapped over the other is unclear. Although quite rare at a rate of $0.01 \%$, we also discovered a number of "double swaps" where both the i7 and i5 indexes from the E. coli libraries were found on human library fragments or vice versa. These double swap reads will not be removed during demultiplexing if they contain an expected combination of indexes, albeit from the wrong sample. In a non-experimental setup, this would manifest as a low rate of sample contamination.
Taken together, these observations indicate that index swapping is occurring relatively uniformly across all libraries within a given pool, that smaller and higher AT content fragments are more likely to be swapped as these are more efficiently amplified by the polymerase, and that this swapping phenomenon has a preference to swap the i5 index side twice as often as the i7 side.

\section{Discussion}

Our findings agree with those reported by others indicating that residual free indexing primer or adapter oligonucleotides carried over into multiplexed library pools can hybridize and extend during the ExAmp clustering chemistry leading to library fragments swapping indexes. This swapping leads to improper demultiplexing, with reads being assigned to the wrong samples which manifests as downstream read contamination in the data. We observe a minimum rate of $0.2 \%$ swapping in ExAmp/ patterned flow cells across all library prep types examined. We propose the only way to effectively eliminate swapped reads from pooled sequencing data is to utilize a non-redundant dual indexing scheme and filter unexpected combinations as even trace amounts of adapter or primer leads to swapping. We believe that the elimination of index swapping is of paramount importance for those performing any sequencing studies.

We observed an average swap rate of $\sim 1 \%$ with a range of 0.2 to $6 \%$ index swapping across our various methods and sequencers, and while this rate of contamination may not affect the ability to trust variant calling for many germline DNA applications, it can lead to spurious results when looking for rare transcripts or 


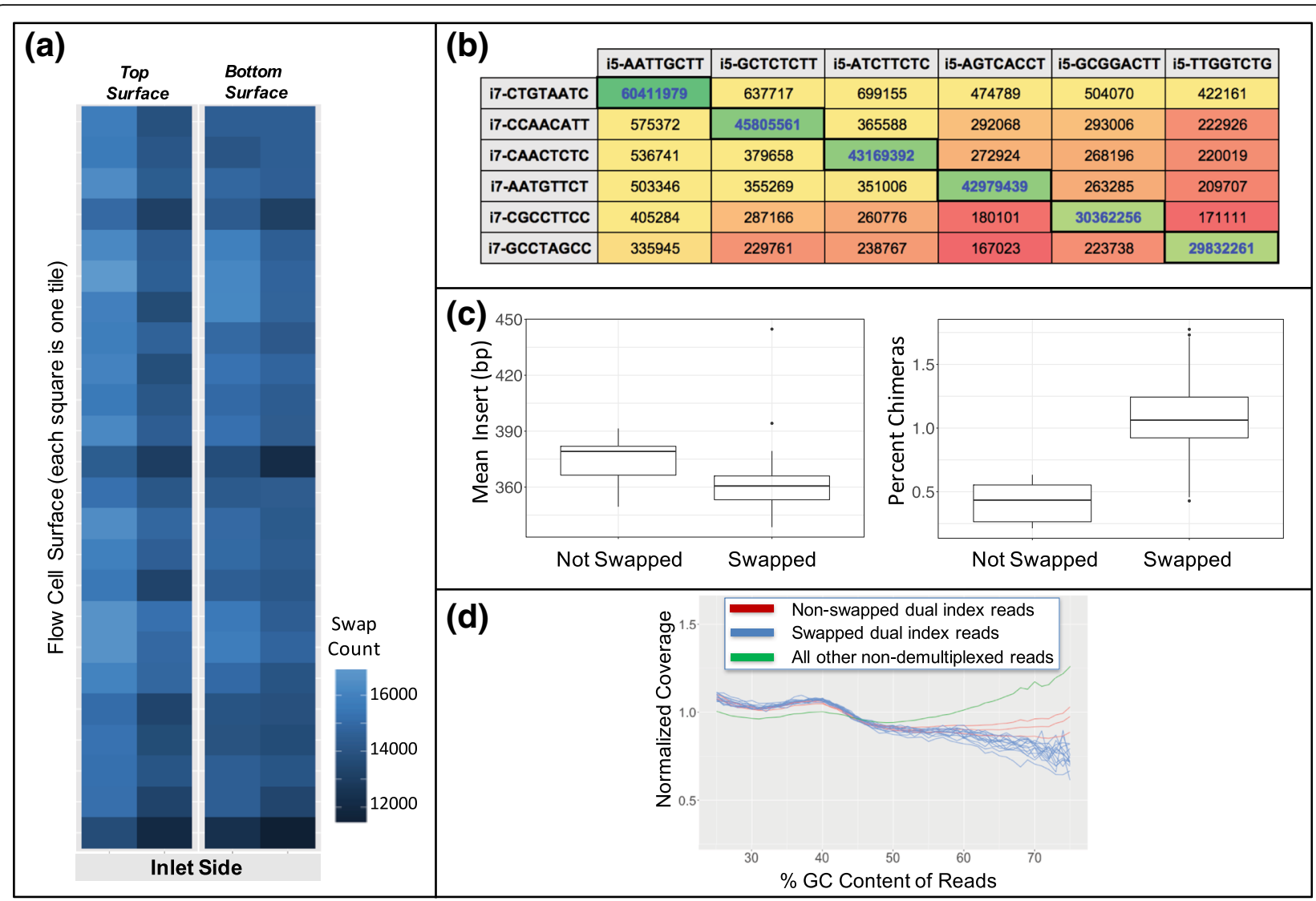

Fig. 5 Characterization of index swapping mechanism. a Diagram of a HiSeqX flow cell lane colored by number of index swaps detected at each surface tile, showing relatively uniform distribution of swapping across the entire lane and both surfaces. b Read counts for all 36 index combinations in a 6-plex pool of uniquely dual indexed libraries. The combinations in heavy bordered cells with blue text along the diagonal are the correct index combinations; read counts for all other combinations are due to index swapping. Note all indexes participate in swapping relatively equally. c Mean insert size (bp) and percent chimerism calculated by Picard for both swapped and non-swapped reads. Swapped reads have shorter inserts and higher rates of chimeric read pairs. $\mathbf{d}$ Normalized human coverage across GC content bins, indicating there are less high GC reads in the swapped population (blue) compared to non-swapped (red) and all other non-demultiplexed (green) populations

Table 2 Swap probability calculations for Human \& E. coli library mixture experiment

\begin{tabular}{|c|c|}
\hline & Read count \\
\hline Total PF indexed reads ${ }^{a}$ & $842,853,260$ \\
\hline Total Non-swapped reads & $807,029,454$ \\
\hline Total swapped reads & $34,219,842$ \\
\hline \multicolumn{2}{|l|}{$p($ Total Swap $)=($ Total swapped reads $) /($ Total PF indexed reads $)=0.0406$} \\
\hline Undetermined i7 or i5 swaps (Human to Human or E. coli to E. coli swaps) & $17,136,498$ \\
\hline Known i7 swaps (Human to E. coli or E. coli to Human swaps) & $5,300,327$ \\
\hline Known i5 swaps (Human to E. coli or E. coli to Human swaps) & $12,697,618$ \\
\hline Known double i7 and i5 swaps (Human to E. coli or E. coli to Human swaps) & 689,363 \\
\hline Estimated total i7 swaps $=\mathrm{i} 7 /(\mathrm{i} 5+\mathrm{i} 7) *$ undetermined $+\mathrm{i} 7+$ double swaps & $110,36,324$ \\
\hline Estimated total i5 swaps $=\mathrm{i} 5 /(\mathrm{i} 5+\mathrm{i} 7) *$ undetermined $+\mathrm{i} 5+$ double swaps & $25,476,845$ \\
\hline \multicolumn{2}{|l|}{ p (i7 Swap) $=($ Estimated total i7) $/($ Non-swap + Undet. + Known i5 + Known i7 + Double $)=0.0131$} \\
\hline $\mathrm{p}($ i5 Swap $)=($ Estimated total i5) $/($ Non-swap + Undet. + Known i5 + Known i7 + Double $)=0.0302$ & \\
\hline
\end{tabular}


fusion events in RNA-seq (as seen in Fig. 3) and in low allele fraction somatic analysis. While mutation callers such as MuTect have filters for many common artifacts that occur during the sequencing process, index swapped reads are unique as they are high quality reads, not errors, that are assigned to the wrong sample [14]. The cancer genomics community has been developing exciting new applications including blood biopsy using circulating tumor DNA (ctDNA), where researchers are trying to detect mutations in ctDNA at allele fractions of $5 \%$ or lower against a background of normal DNA [15-17]. If this level of high sensitivity and confidence is required, then we strongly advocate that unique dual indexing is a necessity as even low rates of sample cross contamination would hinder the accuracy and sensitivity of low allele fraction variant calling.

It should be noted that the phenomenon of index swapping is not restricted to ExAmp chemistry from Illumina, but can occur in any scenario where multiplexed libraries are amplified together in the same vessel and residual adapters and active polymerases are present. In 2013, we had previously observed a similar phenomenon when we began pooling single indexed libraries prior to exome capture (Additional file 1: Figure S2) (previously unpublished data). We observed contamination levels spike, and the rate of this contamination was dependent on what PCR enzyme was used for the pooled capture PCR prior to sequencing. By implementing an early version of unique non-redundant dual indexing, we were able to filter reads with swapped indexes. Today, when we observe the rates of index swapping for exomes, we see higher than expected rates of swapping in exomes even when sequenced on random cluster amplification instruments like the MiSeq (Table 1). When designing sequencing experiments, it is therefore important to keep in mind that that any time samples are amplified together in a pool, whether in a tube during library prep or on a flow cell during ExAmp, there is a danger of index swapping induced cross contamination and non-redundant unique dual indexing should be utilized if possible.

In cases where implementation of dual indexing may be difficult or impossible for a given method, the rate of cross contamination due to index swapping contamination can be measured empirically. We recommend pooling single index libraries made from different organisms if possible, and calculating the rates of reads originating from one organism containing the i7 index from the other. Given that the rates of index swapping can vary so widely from method to method, it may be the case that the swap rate for a given method of interest may be acceptable for the type of analysis being performed and goals of a given project. However, if index swapping is occurring at a higher rate, this may lead to compromised results especially if data is to be used for detecting rare events at low allele fractions or most critically, in clinical settings.

The expected increased sequencing yields from NovaSeq will create a need for pooling larger numbers of samples for many applications to maximize cost efficiency. Major sample preparation reagent providers are just now beginning to offer unique dual indexed adapters and most do not offer full sets of 96, forcing our lab and others to operate in a "do it yourself" mode. There already exists a need for more than 96 samples worth of unique dual indexes for applications such single cell, microbial, or targeted sequencing approaches. Designing and screening new indexes can be a laborious and expensive endeavor for individual labs (see Additional file 3 for suggested design guidelines). As we expanded and optimized our set of dual indexes, we found it necessary to functionally validate each index pair in a costly multistep approach, first screening indexes at the bench to ensure consistent performance in library preparation, and second ensuring that indexes sequenced as expected on multiple models of sequencers. In addition, performing quality control on incoming adapters or primers from oligo synthesis vendors can complicate implementation. Because we have observed quality issues including well to well cross contamination in index plates, we currently perform sequencing-based quality control of all incoming indexing plate batches from our oligo vendor; this is costly but highly recommended step. As the demand for indexing solutions grow, the genomics consumables industry must do better in ensuring that all labs have access to high quality and reliable indexing products that do not require the users to engage in high cost development and quality control testing activities on their own.

\section{Conclusions}

Index swapping in pooled libraries sequenced on Illumina's ExAmp patterned flow cell chemistry is a prevalent phenomenon that can vary in severity but that we observe is always present at a range of $0.2-6 \%$ in sequencing runs on HiSeqX, HiSeq 4000/3000, and NovaSeq. We observe that utilizing unique dual indexes for pooled libraries allows for the removal of swapped reads caused by both multiplex PCR and sequencing-chemistry induced swaps. This is particularly crucial in clinical sequencing settings, single cell sequencing, or analysis of low allele fraction somatic variants where even low percentages of anomalous reads are unacceptable.

The phenomenon of index swapping was discovered and reported by the genomics technology user community, a fact that reinforces the need to be vigilant and closely review any novel sample preparation or sequencing 
technologies from both new and well established companies. We should challenge our technology vendors to ensure they have performed thorough validation of new technologies prior to their release, and further ensure that we have the tools at our disposal to monitor and ensure the integrity of sequencing data.

\section{Methods}

\section{Preparation of sequencing libraries}

Library construction was performed using Kapa Biosystems reagents as described by Fisher et al. [18] with some slight modifications. For whole genomes, initial genomic DNA input was reduced from $3 \mu \mathrm{g}$ to $250 \mathrm{ng}$ for PCR-free or $50 \mathrm{ng}$ for PCR-plus. For germline exomes, input to Nextera based library prep was $50 \mathrm{ng}$. For somatic exomes, DNA input into sheared library prep was $100 \mathrm{ng}$. Subsequent exome capture for both somatic and germline exomes were performed using the Illumina exome oligo pool with a $38 \mathrm{Mb}$ target design. For stranded RNA-seq, 250 ng of total RNA was used as input into the TruSeq stranded mRNA sequencing kit (Illumina). Dual indexed library oligos were customordered from IDT. For ligation adapters, these were ordered HPLC purified, pre-annealed, and in single use plates each at a concentration of $15 \mathrm{uM}$. For Nextera PCR primers, these were ordered standard desalted, forward and reverse premixed, and in single use plates at a concentration of $10 \mathrm{uM}$.

Following sample preparation, libraries were quantified using quantitative PCR (kit purchased from KAPA Biosystems) with probes specific to adapter ends in an automated fashion on Agilent's Bravo liquid handling platform. Based on qPCR quantification, libraries were normalized and pooled on the Hamilton MiniStar liquid handling platform. For HiSeqX and HiSeq4000, pooled samples were normalized to $2 \mathrm{nM}$ and denatured with 0 . $1 \mathrm{~N} \mathrm{NaOH}$ for a loading concentration of $200 \mathrm{pM}$. For Novaseq, pooled samples were normalized $1 \mathrm{nM}$ and denatured with $0.1 \mathrm{~N} \mathrm{NaOH}$ in for a loading concentration of 200 pM. For MiSeq, pooled samples were normalized to $2 \mathrm{nM}$ and denatured with $0.1 \mathrm{~N} \mathrm{NaOH}$ for a loading concentration of $14 \mathrm{pM}$.

\section{Cluster amplification and sequencing}

Cluster amplification of denatured templates and pairedend sequencing was performed according to the manufacturer's protocol (Illumina) for HiSeq X, HiSeq 4000, NovaSeq, or MiSeq. For single index sequencing, an additional 8-bp i7 index read was sequenced. For dual index, additional 8-bp i7 index and 8-bp i5 index reads were sequenced. Dual indexed sequencing on HiSeqX was initially enabled outside of standard control software versions and kits were supplemented with dual index primer "HP14". As of October 2017, Illumina is officially supporting dual indexing on HiSeqX via HiSeq control software v3.5.0 and inclusion of HP14 in revised reagent kits.

\section{Sequencing data analysis}

Output from Illumina software was processed by the Picard data-processing pipeline to yield BAM files containing quality-calibrated, aligned reads. Contamination was calculated using VerifyBamID [9]. Index swapping calculations were made by tabulating per-tile index read information to determine the percentages of both correct and swapped dual-indexed combinations present.

For experiments comparing metrics for swapped versus non-swapped reads, 12 libraries of NA12878 human DNA and 12 libraries of E. coli-K12_MG12655 were prepared as PCR-free genome libraries with unique dual index combinations. The Picard data-processing pipeline was then used to aggregate BAM files while allowing all possible barcode combinations. Since there are 24 possible barcodes (for both i 5 and i7), there are $24^{\wedge} 2=576$ possible pairs of barcodes. Each possible barcode pair was aggregated into its own BAM file resulting in 576 BAM files, of which 552 constitute swaps. We then aligned these BAMs to a reference containing both human and $E$. coli contigs and counted the number of reads mapping to human and E. coli respectively from each file. Insert size, the rate of chimerism, and GC content were then calculated independently for the swapped and non-swapped BAMs.

We assume that all reads were mapped correctly to their organism of origin, and from this we are able to determine the location where a swap occurred, i5 or i7 index. When reads and barcodes belong to the same species we can identify that a swap has occurred, however we cannot determine where the swap occurred. When barcodes originally belonging to differing organisms are found on a read, we can identify the barcode that swapped by assuming that the unique barcode belonging to the organism the read maps to did not swap. Likewise, it is possible to identify cases where both the i5 and i7 barcodes swapped when a read maps to a particular organism, but both its barcodes point to a sample from the other organism. Using this simple approach we were able to estimate the probability of swaps occurring at each end of the library fragment.

\section{Additional files}

Additional file 1: Figure S1. Initial testing of single vs dual indexed sequencing on HiSeq. Figure S2. Previous experience with index swapping during exome capture library preparation (previously unpublished data). (DOCX $1113 \mathrm{~kb}$ )

Additional file 2: A listing 192 indexes validated at Broad Genomics across multiple library methods and sequencer models. (XLSX 17 kb) 
Additional file 3: Suggested guidelines for designing robust indexes for Illumina sequencing. (DOCX $129 \mathrm{~kb}$ )

\section{Abbreviations}

BAM: Binary Alignment/Map; ctDNA: Circulating tumor DNA;

ExAmp: Exclusion amplification; i5: Illumina's acronym for the second index read; i7: Illumina's acronym for the first index read; Q30: Phred scaled sequencing quality score of 30

\section{Acknowledgements}

We thank the rest of the Broad Genomics Platform for generating all data used in this study.

\section{Funding}

This study was partially funded by the Center for Common Disease Genetics (grant ID UM1HG008895).

\section{Availability of data and materials}

This study involved aggregation of meta data and calculated sequencing metrics across many thousands of samples sequenced over a two year period. However, a subset of PCR-free human genome data used in this article can be found in the gnomAD genome aggregation database (http://gnomad.broadinstitute.org). The remainder of the sequence data referenced here are restricted due to third party obligations.

\section{Authors' contributions}

MC provided technical and intellectual contributions, and prepared manuscript text, figures, and tables. MF performed all swap characterization computational analyses, made technical and intellectual contributions, and reviewed manuscript. LG and BG generated the RNA-seq fusion contamination data and reviewed abstract. JA, YF, SF, LH, TH, TM, GV, MD, WB, TD, SD, NJL, and SG all made experimental, technical, and/or intellectual contributions and reviewed the manuscript. All authors have read and approved the manuscript prior to submission.

\section{Ethics approval and consent to participate}

Only sequencing metric values automatically calculated by the Picard analysis pipeline (\% contamination, etc.) and library index read data (\% demultiplexed reads, \% index swapping, etc.) were examined. For E. coli mixture study, human DNA used was obtained from Coriell Biorepository (NA12878) and was consented for research.

\section{Competing interests}

The authors declare that they have no competing interests.

\section{Publisher's Note}

Springer Nature remains neutral with regard to jurisdictional claims in published maps and institutional affiliations.

Received: 23 November 2017 Accepted: 19 April 2018

Published online: 08 May 2018

\section{References}

1. Shen MR, Boutell JM, Stephens KM, Ronaghi M, Gunderson K, Venkatesan BM, Bowen MS, Vijayan K. Kinetic exclusion amplification of nucleic acid libraries. USPTO 20160053310:A1. US Patent, filed October 9, 2015, and issued February 25, 2016.

2. Illumina, Inc. Illumina HiSeqX series specification sheet. 2017. https:// www.illumina.com/content/dam/illumina-marketing/documents/ products/datasheets/datasheet-hiseq-x-ten.pdf. Accessed 15 Nov 2017.

3. Illumina, Inc. Illumina NovaSeq specification sheet. 2017. https://www. illumina.com/content/dam/illumina-marketing/documents/products/ datasheets/novaseq-6000-system-specification-sheet-770-2016-025.pdf Accessed 15 Nov 2017.

4. Sinha R, Stanley G, Gulati GS, Ezran C, Travaglini K, Wei E, et al. Index switching causes 'spreading-of-signal' among multiplexed samples in illumina HiSeq 4000 DNA sequencing. bioRxiv. 2017; https://doi.org/10. $1101 / 125724$.

5. Illumina, Inc. Effects of index Misassignment on multiplexing and downstream analysis. 2017. https://www.illumina.com/content/dam/ illumina-marketing/documents/products/whitepapers/index-hopping-whitepaper-770-2017-004.pdf?linkld=36607862. Accessed 15 Nov 2017.

6. Griffiths JA, ATL L, Richard AC, Bach K, Marioni JC. Detection and removal of barcode swapping in single-cell RNA-Seq data. bioRxiv. 2017; https://doi. org/10.1101/177048.

7. Vodak D, Lorenz S, Nakken S, Aashein LB, Holte H, Bai B, Myklebost, O, Meza-Zepeda, LA, Hovig, E. Sample-index misassignment impacts tumor exome sequencing. bioRxiv. 2017. doi.org:https://doi.org/10.1101/182659 9.

8. Van der Valk T, Vezzi F, Ormestad M, Dalen L, Guschanski K. Low rate of index hopping on the illumina HiSeq X platform. bioRxiv. 2017; https://doi. org/10.1101/179028.

9. Jun G, Flickinger M, Hetrick KN, Romm JM, Doheny KF, Abecasis GR, Boehnke M, Kang HM. Detecting and estimating contamination of human DNA samples in sequencing and array-based genotype data. Am J Hum Genet. 2012:91(5):839-48.

10. Owens GL, Todesco M, Drummond EBM, Yeaman S, Rieseberg LH. A novel post hoc method for detecting index switching finds no evidence for increased switching on the Illumina HiSeq X. Mol Ecol Resour. 2017;00:1-7. https://doi.org/10.1111/1755-0998.12713.

11. Larsson AJ, Stanley G, Sinha R, Weissman IL, Sandberg R. Computational correction of cross-contamination due to exclusion amplification barcode spreading. bioRxiv. 2017; https://doi.org/10.1101/176537.

12. Kircher M, Sawyer S, Meyer M. Double indexing overcomes inaccuracies in multiplex sequencing on the Illumina platform. Nucleic Acids Res. 2012; 40(1):e3.

13. Shagin DA, Lukyanov KA, Vagner LL, Matz MV. Regulation of average length of complex PCR product. Nucleic Acids Res. 1999;27(18):e23.

14. Cibulskis K, Lawrence MS, Carter SL, Sivachenko A, Jaffe D, Sougnez C, Gabriel S, Meyerson M, Lander ES, Getz G. Sensitive detection of somatic point mutations in impure and heterogeneous cancer samples. Nat Biotechnol. 2013;31(3):213-9.

15. Wan JCM, Massie C, Garcia-Corbacho J, Mouliere F, Brenton JD, Caldas C, Pacey S, Baird R, Rosenfeld N. Liquid biopsies come of age: towards implementation of circulating tumour DNA. Nat Rev Cancer. 2017;17:223-38.

16. Stover DG, Parsons HA, Ha G, Freeman SS, Barry WT, Guo H, Choudhury AD, Gydush G, Reed SC, Rhoades J, Rotem D, Hughes ME, Dillon DA, Partridge AH, Wagle N, Krop IE, Getz G, Golub TR, Love JC, Winer EP, Tolaney SM, Lin $\mathrm{NU}$, Adalsteinsson VA. Association of Cell-Free DNA tumor fraction and somatic copy number alterations with survival in metastatic triple-negative breast Cancer. J Clin Oncol. 2018;36:543-53. https://doi.org/10.1200/JCO. 2017.76 .0033$.

17. Xu C, Nezami Ranjbar MR, Wu Z, DiCarlo J, Wang Y. Detecting very low allele fraction variants using targeted DNA sequencing and a novel molecular barcode-aware variant caller. BMC Genomics. 2017:18(1):5.

18. Fisher S, Barry A, Abreu J, Minie B, Nolan J, Delorey TM, et al. A scalable, fully automated process for construction of sequence-ready human exome targeted capture libraries. Genome Biol. 2011;12(1):R1. https://doi.org/10.1186/gb-2011-12-1-r1.

\section{Ready to submit your research? Choose BMC and benefit from:}

- fast, convenient online submission

- thorough peer review by experienced researchers in your field

- rapid publication on acceptance

- support for research data, including large and complex data types

- gold Open Access which fosters wider collaboration and increased citations

- maximum visibility for your research: over $100 \mathrm{M}$ website views per year

At BMC, research is always in progress.

Learn more biomedcentral.com/submissions 